\title{
Die Macht der Erwartungen
}

\author{
Winfried Rief \\ Klinische Psychologie und Psychotherapie, Philipps-Universität Marburg, Deutschland
}

Einem Sprichwort zufolge soll der Glaube Berge versetzen können. Diese Annahme hat auch in der Psychologie zur Entwicklung zahlreicher Theorien geführt, am prominentesten sicherlich die Theorie der «Self-fulfilling Prophecy». Gerade auch in der Verhaltenstherapie, wo wir uns besonders mit der Entwicklung von Methoden zur Selbstregulation beschäftigen [Forstmeier und Rüddel, 2005], sollten wir die Frage stellen, welche Erwartungen wann durch welche Verhaltensweisen von Therapeuten induziert werden und welche Effekte dies haben kann. Stimmt es wirklich, dass Erwartungen, zumindest bildlich, Berge versetzen können?

Die neuere Forschung zur Rolle von Erwartungen im klinischen Kontext bestätigt zwar nicht, dass sich durch Erwartungen Berge bewegen, es lassen sich jedoch zahlreiche klinische Phänomene substantiell beeinflussen. Gerade auch die Möglichkeiten der funktionellen Bildgebung machen deutlich, dass Erwartungen nicht reine «Geistesphänomene» sind, sondern direkt Relevanz für Hirnaktivierungsprozesse haben. So konnten Lorenz et al. [2005] zeigen, dass Menschen, denen unterschiedlich intensive Schmerzreize zugefügt werden, ein komplexes Muster elektrophysiologischer und elektromagnetischer Reaktionen im Gehirn produzieren. Werden für unterschiedliche Schmerzreize kurz vorher unterschiedliche Warnreize dargeboten, kann der Effekt von Erwartungshaltungen genauer untersucht werden. So konnten die Autoren zeigen, dass die Hirnaktivität bei der Erwartung von Schmerzreizen (die dann aber ausblieben) fast identisch war mit der Hirnaktivität, wenn reale Schmerzreize appliziert wurden. Wurde ein starker Schmerzreiz erwartet, war die Hirnaktivität sogar größer, als wenn ein schwächerer Schmerzreiz real appliziert wurde. Dies macht deutlich, dass die Erwartung eines Schmerzerlebens (z.B. bei Patienten mit chronischen Schmerzen) dazu führt, dass das Hirn in einen Aktivierungszustand gebracht wird, der die Wahrnehmung von Schmerzen deutlich erleichtert bzw. sogar verstärkt.
Im klinischen Kontext sind die Effekte von Erwartungshaltungen am besten mit Placebo-Designs untersucht worden. Placebos können sowohl schmerzreduzierende als auch symptomprovozierende Effekte haben. So berichten z.B. Personen in klinischen Studien regelmäßig von Beschwerden, die sie als Nebenwirkungen der Medikamente interpretieren, obwohl diese Personen in der Placebo-Gruppe waren, also gar kein Medikament erhalten haben (Nocebo-Effekt) [z.B. Barsky et al., 2002; Rief et al., 2006].

Werden Schmerzreize tatsächlich appliziert, ergibt sich im Gehirn ein typisches Aktivierungsmuster der sogenannten «Schmerzmatrix» (z.B. somatosensorische Felder S1, S2, Inseln, Thalamus, zum Teil auch Amygdala, präfrontaler Cortex und Anteriores Cingulum). Wird ein solcher Schmerzreiz unter Placebo-Induktion appliziert, ist die entsprechende Aktivierung im Gehirn auch in den somatosensorischen Feldern deutlich geringer und das subjektive Schmerzempfinden niedriger [z.B. Price et al., 2006; Bingel et al., 2006]. Gerade neurophysiologisch erscheint hierbei die Studie von Bingel et al. besonders interessant. Personen bekamen auf beide Handrücken Handcremes appliziert, wobei in einem Fall erläutert wurde, dass es sich um eine schmerzlindernde Creme handele («Placebo-Instruktion») und im anderen Fall eine neutrale Erläuterung über den Zweck der Creme gegeben wurde. Anschließend wurde mit einem Laserstrahl ein Schmerzreiz auf den Handrücken appliziert. Unter der neutralen Bedingung fand sich in den meisten Hirnarealen eine größere Hirnaktivität als unter der Placebo-Instruktion. Interessanterweise ergab sich in einem Hirnbereich aber auch unter der PlaceboBedingung eine erhöhte Aktivität: Im rostralen Teil des anterioren cingulären Cortex scheint sich gerade beim Versuch der Bewältigung von Beschwerden durch positive Erwartungen mehr Aktivität abzuspielen [Lorenz et al., 2005]. Von den Autoren wird dies dahingehend interpretiert, dass dieses Hirnareal für die Kontrolle von Schmerzempfindungen zuständig

\begin{tabular}{llll}
\hline KARGER & ๑) 2007 S. Karger GmbH, Freiburg & & Prof. Dr. Winfried Rief \\
Fax +49 7614520714 & Accessible online at: & & Philipps-Universität Marburg \\
$\begin{array}{l}\text { E-mail Information@Karger.de } \\
\text { www.karger.com }\end{array}$ & www.karger.com/ver & Klinische Psychologie und Psychotherapie \\
& & Gutenbergstr. 18, 35032 Marburg, Deutschland \\
& & Tel. +49 6421 282-3657, Fax -8904 \\
& & E-mail rief@staff.uni-marburg.de
\end{tabular}


ist, indem es z.B. niedriger liegende Hirnstrukturen (z.B. Amygdala) hemmt und dadurch eine Filterfunktion ausübt. Diese nun auch neurophysiologisch besser belegten Möglichkeiten von Erwartungen können positiv genutzt werden, führen bei Missachtung jedoch oft auch zu negativen Effekten oder zumindest zum Verlust therapeutischer Möglichkeiten. Gerade im klinischen Kontext zeigt sich doch oftmals, dass negative Erwartungen induziert werden und Personen diese über einen längeren Zeitraum beibehalten. Wird Patienten z.B. mitgeteilt, dass eine bestimmte organische Untersuchung durchgeführt werden muss, da vielleicht eine schwere körperliche Erkrankung die bestehenden Beschwerden erklärt, wird der Patient mindestens bis zur Durchführung dieser Untersuchung in einer eher negativen Erwartungshaltung sein. In einer eigenen Studie in Zusammenarbeit mit der Universität Auckland in Neuseeland wurde deshalb versucht, diese negative Erwartungshaltung möglichst gering zu halten. Personen, die zu einem Belastungs-EKG angemeldet wurden, wurde deshalb frühzeitig (vor der Untersuchung) mitgeteilt, dass das wahrscheinlichste Ergebnis des Belastungs-EKGs sein werde, dass der Herzmuskel gesund und die bestehenden Herzbeschwerden auf andere, weniger dramatische Ursachen zurückzuführen sind. Erhielten Personen diese «A priori Beruhigung» frühzeitig, so entwickelten sie nach der Untersuchung weniger Herzbeschwerden und glaubten ihren Ärzten auch eher, wenn diese ihnen mitteilen, dass die Untersuchung keinen pathologischen Befund erbracht hatte [Petrie et al., 2007]. Erfolgte diese «A priori Beruhigung» nicht, so glaubten 50\% der Patienten ihren Ärzten nicht oder nur unvollständig, dass das EKG wirklich ohne pathologischen Befund und ihr Herzmuskel gesund wäre.

Diese und ähnliche Ergebnisse sollten Anlass für uns alle sein, das gesamte Gesundheitssystem, aber speziell auch unsere Interaktionsmuster mit Patienten zu überdenken und auf die Induktion von negativen Erwartungshaltungen zu prüfen. Je länger Patienten negative oder unsichere Erwartungshaltungen z.B. vor medizinischen Untersuchungen haben, desto stärker werden Wahrnehmungsprozesse für Symptome gefördert (vgl. das Interview mit Barsky in diesem Heft). Die Beeinträchtigungen durch die Beschwerden werden stärker und die Rückkehr zu einem normalen Alltag wird erschwert. Wird einem Suchtpatienten mitgeteilt, dass er wahrscheinlich erst richtig «abstürzen» muss, bevor eine Umkehr möglich ist, so wird damit dieser negative Verlauf gebahnt und vorbereitet. Wacht der Hypochondrie-Patient morgens mit dem Gedanken auf, ob sein Kehlkopf wirklich gesund ist oder nicht doch Kehlkopfkrebs vorliegt, wird er dies vielleicht durch mehrere Schluckübungen prüfen und damit sein Gehirn in eine Wahrnehmungsbereitschaft für Missempfindungen im Kehlkopfbereich bringen.

Durch diese Ergebnisse wird deutlich, dass wir auch psychotherapeutische Interventionen daraufhin untersuchen sollten, ob sie ihr Potential, entsprechende positive Erwartungshaltungen aufzubauen, auch ausreichend nutzen. Dies gilt für Behandlungsprogramme für Krebspatienten [z.B. Zimmermann et al., 2006], aber natürlich ganz besonders auch für depressive Patienten, bei denen eine negative Erwartungshaltung direkt zur Symptomatik gehört [z.B. Risch und Stangier, 2006]. Bei allen chronischen Erkrankungen (z.B. chronische Schmerzsyndrome) liegt es nahe, dass viele Patienten eine verfestigte Meinung über einen weiteren negativen Verlauf haben. In allen diesen Fällen wäre es sinnvoll, die Auswirkungen dieser negativen Erwartungshaltungen mit den Patienten zu reflektieren und Möglichkeiten auszuloten, durch bessere Erwartungshaltungen auch mehr therapeutischen Erfolg zu haben. Wahrscheinlich können Erwartungshaltungen wirkliche Berge nur selten versetzen. Aber ein verbesserter Einsatz des Potentials von Erwartungen sowie eine Reduktion der schädigenden Auswirkungen negativer Erwartungen können auch in der Psychotherapie zu einer deutlichen Verbesserung beitragen.

\section{Literatur}

Barsky AJ, Saintfort R, Rogers MP, Borus JF: Nonspecific medication side effects and the nocebo phenomenon. JAMA 2002;287:622-626.

Bingel U, Lorenz J, Schoell E, Weiller C, Büchel C: Mechanisms of placebo analgesia: rACC recruitment of a subcortical antinociceptive network. Pain 2006; 120:8-15.

Forstmeier S, Rüddel H: Zur Überlegenheit von Selbstregulation über Selbstkontrolle in Psychotherapie und psychosomatischer Rehabilitation. Verhaltenstherapie 2005;15:158-167.
Lorenz J, Hauck M, Paur RC, Nakamura Y, Zimmermann R, Bromm B, Engel AK: Cortical correlates of false expectations during pain intensity judgments $-\mathrm{a}$ possible manifestation of placebo/nocebo cognitions. Brain Behav Immun 2005;19:283-295.

Petrie K, Müller T, Schirmbeck F, Donkin L, Broadbent E, Ellis CJ, Gamble G, Rief W: Effect of providing information about normal test results on patients' reassurance: randomised controlled trial. BMJ 2007;334:354-357.

Price DD, Craggs J, Verne GN, Perlstein WM, Robinson ME: Placebo analgesia is accompanied by large reductions in pain-related brain activity in irritable bowel syndrome patients. PAIN 2006;124:238-239.
Rief W, Avorn J, Barsky AJ: Medication-attributed adverse effects in placebo groups. Implications for assessment of adverse effects. Arch Intern Med 2006;166: 155-160.

Risch AK, Stangier U: Neuere kognitiv-verhaltenstherapeutische Ansätze zur Rückfallprävention bei rezidivierender Depression. Verhaltenstherapie 2006;16: 275-282.

Zimmermann T, Heinrichs N, Scott JL: CanCope «Schritt für Schritt». Die Effektivität eines partnerschaftlichen Unterstützungsprogramms bei Frauen mit Brust- oder gynäkologischen Krebserkrankungen. Verhaltenstherapie 2006;16:247-256. 